\title{
Dieta cetogénica como tratamiento de la epilepsia refractaria durante la infancia: revisión práctica de la literatura
}

\author{
Santiago Vásquez-Builes ${ }^{1,2}$, Paulina Jaramillo-Echeverri ${ }^{1,2}$, Laura Montoya-Quinchia ${ }^{1,2}$ y \\ Laura Apraez-Henao ${ }^{2}$
}

${ }^{1}$ Facultad de Medicina; ${ }^{2}$ Semillero de neurociencias, Universidad CES; ${ }^{3}$ Facultad de Medicina, Fundación Universitaria San Martin, Sabaneta. Medellin, Colombia

\begin{abstract}
Resumen
La epilepsia es una enfermedad neurológica grave y de evolución crónica que afecta tanto a niños como a adultos. Desde los años veinte, la dieta cetogénica (DC) se ha considerado una opción terapéutica en pacientes con epilepsia refractaria y actualmente se utiliza como primera línea de tratamiento para algunos trastornos metabólicos. La DC puede ser eficaz no solo en epilepsias generalizadas o multifocales sintomáticas, sino también en focales y lesiónales. Su acción antiepiléptica se basa en la teoría de que los cuerpos cetónicos, sintetizados en el hígado a partir de ácidos grasos de cadena larga y media, funcionan como anticonvulsivos directos al cruzar la barrera hematoencefálica. El presente artículo realiza una descripción práctica y detallada de la literatura médica sobre las indicaciones, contraindicaciones, aspectos clínicos, fisiológicos y terapéuticos de la $D C$, con el objetivo de brindar a la comunidad médica las habilidades necesarias para un cuidado integral de los niños candidatos a DC o que actualmente la utilizan como tratamiento de su epilepsia refractaria.
\end{abstract}

Palabras clave: Dieta cetogénica. Epilepsia refractaria. Infancia. Epilepsia en pediatría.

\section{Ketogenic diet as a treatment for refractory epilepsy during infancy: A practical literature review}

\begin{abstract}
Epilepsy is a severe neurological illness disease of chronic evolution that affects both children and adults. Since the 1920s, the cetogenic diet has been considered as a therapeutic option in patients with refractory epilepsy and is currently considered as the first line of treatment for some metabolic disorders. The cetogenic diet can be effective in generalized seizures but also in focal seizures. Its antiepileptic actions are based on the fact that the ketone body that are sintetized in the liver from long and medium chain fatty acid work as direct anticonvulsant as they cross the hood-brain barrier. This paper presents a detailed and practical description of the state of the current medical literature of the indications, contraindications, clinical, physiological and therapeutical aspects of the cetogenic diet in order to provide to the medical community the required habilities to take proper care of prospect children for a cetogenic diet or children who are already under it as treatment for their refractory epilepsy.
\end{abstract}

Key words: Ketogenic diet. Refractary epilepsy. Childhood. Epilepsy in pediatrics.

Correspondencia:

Santiago Vásquez-Builes

E-mail: santiagovasquezb @ hormail.com
Fecha de recepción: 09-09-2018

Fecha de aceptación: 28-12-2018

DOI: 10.24875/RMN.M19000022
Disponible en internet: 20-03-2019

Rev Mex Neuroci. 2019;20(1):14-20

www.revmexneurociencia.com

1665-5044/@ 2019. Academia Mexicana de Neurología A.C. Publicado por Permanyer México. Este es un artículo Open Access bajo la licencia CC BY-NC-ND (http://creativecommons.org/licenses/by-nc-nd/4.0/). 


\section{Introducción}

La epilepsia es una enfermedad neurológica grave y de evolución crónica ${ }^{1}$. Se estima que afecta aproximadamente a 40 millones de personas en el mundo. Las personas que la padecen tienen una tasa de muerte mayor respecto a la población general. Tienen la enfermedad 10.5 millones de niños menores de 15 años, lo cual representa el $25 \%$ de la población con esta condición ${ }^{2}$. Su incidencia anual oscila entre 41 a 187 casos por cada 100,000 niños, sin embargo, existe una gran variación de esta entre los resultados obtenidos en diversos estudios epidemiológicos debido a múltiples factores; no obstante, la diferencia principal tiene que ver con la metodología de los estudios ${ }^{1,3}$.

La Liga Internacional contra la Epilepsia (ILAE por sus siglas en inglés) define la epilepsia como: 1) presencia de dos o más convulsiones no provocadas con 24 horas de separación entre el primer y segundo evento convulsivo; 2) convulsión no provocada y una probabilidad de repetir la convulsión $\geq 60 \%$, y 3) diagnóstico de un síndrome epiléptico ${ }^{4}$.

Desde la fisiopatología, la epilepsia se produce por impulsos nerviosos anormales y excesivos de un grupo de neuronas. Esto es explicado principalmente por pérdida del equilibrio entre inhibición y excitación en todo 0 alguna parte del cerebro ${ }^{5}$.

Los impulsos nerviosos son normalmente controlados por neurotransmisores. El principal neurotransmisor inhibitorio (ácido $\gamma$-aminobutírico, GABA por sus siglas en inglés) y el excitatorio (glutamato) pueden inhibir o activar el impulso nervioso respectivamente. El desequilibrio de ambos lleva a la aparición clínica de crisis epilépticas. Las características de las crisis son variables y dependen de la zona del cerebro en la que empieza el trastorno y de su propagación ${ }^{5}$.

En el diagnóstico reciente de epilepsia, la primera línea de tratamiento son los anticonvulsivantes ${ }^{6}$. Cuando no hay respuesta a tres medicamentos anticonvulsivos se denomina epilepsia refractaria ${ }^{7}$.

En niños con epilepsia refractaria y un foco de crisis resecable, la cirugía con resección dirigida del foco convulsivo es una opción viable de tratamiento, sin embargo, en pacientes que no son candidatos al procedimiento quirúrgico la dieta cetogénica (DC) se convierte en la mejor opción terapéutica ${ }^{8}$.

La DC es la modalidad antiepiléptica más potente entre los tratamientos no farmacológicos y no quirúrgicos. Se ha utilizado como tratamiento para la epilepsia durante varias décadas y es la primera línea de tratamiento para algunos trastornos metabólicos, como en la deficiencia del transportador de glucosa $1^{8}$. Actualmente se ha demostrado que la DC puede ser eficaz no solo en epilepsias generalizadas o multifocales sintomáticas, sino también en parciales y lesionales. Los niños menores de doce años parecen generar cetonas con mayor facilidad que los adolescentes y adultos, sin embargo, la dieta ha sido efectiva en todas las edades. Su eficacia en la epilepsia refractaria oscila entre el 30 y el $50 \%$ de los casos $^{9}$.

El mecanismo de acción por el cual la DC funciona como antiepiléptico aún permanece en investigación. La principal teoría se basa en que los cuerpos cetónicos (acetoacetato, acetona y beta-hidroxibutirato), sintetizados en el hígado a partir de ácidos grasos de cadena larga y media, son anticonvulsivos directos al cruzar la barrera hematoencefálica. Al ingresar al cerebro funcionan como fuente primaria de energía e inducen un estado de cetosis ${ }^{10,11}$. Durante la cetosis hay mayor eliminación de glutamato, disminución de su transporte vesicular y aumento de la conversión de este neurotransmisor a GABA ${ }^{12}$.

El objetivo del presente artículo es realizar una descripción práctica y detallada de la literatura médica sobre las indicaciones, contraindicaciones, aspectos clínicos, fisiológicos y terapéuticos de la DC, con el objetivo de brindar a la comunidad médica las habilidades necesarias para un cuidado integral de los niños candidatos a DC o que actualmente la utilizan como tratamiento de su epilepsia refractaria.

Se resumen artículos publicados en los últimos 30 años encontrados por una búsqueda en la literatura, usando las bases de datos de PubMed, ClinicalKey y Elsevier. Los términos «ketogenic diet», «refractary epilepsy», "childhood» $\mathrm{y}$ «epilepsy in pediatrics» se buscaron solos y en combinación. Se tuvieron en cuenta artículos en inglés y en español.

\section{Evidencia epidemiológica de la dieta cetogénica}

La DC debe ser considerada una alternativa segura y eficaz para el tratamiento de la epilepsia refractaria ${ }^{13}$. Se ha demostrado que aproximadamente la mitad de los pacientes logran una disminución de un $50 \%$ en el número de convulsiones y cerca de un tercio una disminución del $90 \%{ }^{14}$.

En un estudio realizado con 23 pacientes entre los 5 meses y los 2 años de edad, con un periodo de observación durante los 3, 6, 9 y 12 meses desde el inicio de la dieta, determinó que existe una disminución de 
los episodios convulsivos en un $38,39,53$ y $46 \%$ al iniciar la $\mathrm{DC}^{14}$.

Un estudio prospectivo realizado en Turquía entre el año 2012 y el año 2016, con una muestra de 389 pacientes con epilepsia refractaria, demostró que quienes recibieron manejo con DC durante 1, 3, 6 y 12 meses tuvieron como resultado una respuesta al tratamiento del $65.8 \%$ al mes, el $74.7 \%$ a los 3 meses, el $70.6 \%$ a los 6 meses y el $83.1 \%$ a los 12 meses. Ninguno de los niños tuvo una mayor frecuencia de ataques ${ }^{15}$.

Por otro lado, un estudio retrospectivo publicado en el año 2018 en la revista Pediatric Neurology, con una muestra de 27 pacientes con epilepsia refractaria, demostró que todos los pacientes al iniciar una DC tradicional presentaron una tasa de respuesta a los 1, 6 y 12 meses del 68, 82 y $91 \%$ respectivamente, logrando cese total de los episodios convulsivos en un 20, 29 y $27 \%$, respectivamente ${ }^{16}$.

La dieta se ha visto que es particularmente efectiva en espasmos infantiles y mioclonías. Además, no solamente disminuye la frecuencia de las convulsiones, sino que mejora el comportamiento y la funcionalidad de los pacientes ${ }^{17}$.

\section{Mecanismo de acción de la dieta cetogénica}

La DC se caracteriza por tener alto contenido de grasas, niveles adecuados de proteínas $(1 \mathrm{~g} / \mathrm{kg})$ y bajo contenido de carbohidratos. Por su composición, al consumirse produce en el organismo cambios metabólicos asociados con el estado de inanición. Cuando se inicia la DC, durante las primeras horas ocurren cambios en los niveles de cetonas plasmáticas, la insulina, la glucosa, el glucagón y los ácidos grasos libres ${ }^{18}$.

La DC se basa en la teoría que los cuerpos cetónicos (acetoacetato, acetona y beta-hidroxibutirato), sintetizados en el hígado a partir de ácidos grasos de cadena larga y media, son anticonvulsivos directos al cruzar la barrera hematoencefálica ${ }^{10}$.

Se ha demostrado que la biogénesis mitocondrial aumentada, la fosforilación oxidativa, el aumento de los niveles de GABA, la disminución en la excitabilidad neuronal y la estabilización en la función sináptica se producen en pacientes con DC ${ }^{18,19}$. La disminución de la transmisión sináptica glutamatérgica y su conversión a GABA también se han asociado con la dieta ${ }^{12,20}$.

En el momento, se desconoce cuál de estos cambios metabólicos es el principal responsable de la mejoría clínica en la frecuencia de las convulsiones.
Tabla 1. Indicaciones de la dieta cetogénica

\section{Indicaciones de la dieta cetogénica}

1. Falla en el control anticonvulsivo luego de la tercera medicación

2. Epilepsia médicamente refractaria

3. Síndromes epilépticos

- Epilepsia mioclónica astática

- Convulsiones del complejo de esclerosis tuberosa

- Síndrome de West con refractariedad a vigabatrin u

hormona adrenocorticotropa

- Síndrome de Dravet

4. Epilepsias sintomáticas

- Enfermedad de cuerpos de Lafora

- Convulsiones del síndrome de Rett

- Síndrome de Landau-Kleffner

- Panencefalitis esclerosante subaguda

- Encefalopatía epiléptica relacionada con infecciones febriles

- Estatus convulsivo refractario

5. Tratamiento de primera línea para

- Deficiencia del transportador de glucosa de tipo 1

- Deficiencia de piruvato deshidrogenasa

6. Enfermedades metabólicas

- Deficiencia de fosfofructocinasa

- Glucogenosis tipo V

- Trastornos del complejo respiratorio mitocondrial

\section{Indicaciones de la dieta cetogénica}

La indicación de la dieta está a cargo de un neurólogo pediatra, quien determina qué pacientes son candidatos a este tratamiento ${ }^{21,22}$. La decisión de iniciar la dieta o no debe tomarse después de realizar una evaluación integral del paciente y de haber analizado los factores de riesgo y la posibilidad de aparición de efectos adversos al inicio de la dieta ${ }^{23}$.

Las indicaciones de DC son muy variables (Tabla 1). La selección y la evaluación correcta del paciente son fundamentales para lograr el objetivo buscado $0^{7,24-26}$.

\section{Contraindicaciones de la dieta cetogénica}

Previo al inicio de la DC es necesario descartar situaciones clínicas y sociales en las que la dieta está contraindicada (Tabla 2) ${ }^{10,23,27}$.

\section{Preparación para la dieta}

Es fundamental que previo al inicio de la DC se evalúe la presencia de una serie de prerrequisitos que van a favorecer la máxima seguridad y efectividad de la dieta. Estos se basan principalmente en la evaluación neurológica, pediátrica y nutricional9: 
Tabla 2. Contraindicaciones de la dieta cetogénica Contraindicaciones de la dieta cetogénica

1. Absolutas

- Trastornos del transporte de los ácidos grasos

- Trastornos de la oxidación de los ácidos grasos (betaoxidación)

- Deficiencia de piruvato carboxilasa

- Porfiria

- Desnutrición moderada o grave

2. Relativas

- Estructura psicológica familiar inestable

- Epilepsias con claro foco quirúrgico determinado con neuroimagen y monitoreo videoelectroencefalográfico

- En la evaluación neurológica se debe realizar una historia clínica completa donde se determine el tipo de epilepsia, frecuencia, etiología, respuesta a los anticonvulsivantes y el estado neurocognitivo. Además se deben solicitar ayudas diagnósticas como un electroencefalograma, una polisomnografía y una resonancia magnética cerebral, con el objetivo de determinar la susceptibilidad a tratamiento quirúrgico. Se recomienda añadir un perfil metabólico completo que incluya la medición de ácidos orgánicos en orina, aminoácidos en suero, amonio, ácido láctico y acilcarnitina sérica para descartar encefalopatías metabólicas en pacientes donde no es clara la etiología ${ }^{28}$.

- En la evaluación pediátrica se debe descartar la presencia de otras comorbilidades que se puedan exacerbar con la dieta. Según esto, se recomienda la realización de una ecocardiografía, principalmente si el paciente tiene historia de una cardiopatía. Se debe realizar una ecografía abdominal para descartar la presencia cálculos renales o hígado graso, hallazgos sugestivos de trastornos metabólicos. Además, se sugiere complementar con paraclínicos como hemoleucograma, función hepática, función renal, perfil lipídico, glucemia, ionograma, gases arteriales, vitamina $\mathrm{D}$, hormona paratiroidea, osteocalcina, uroanálisis, creatinina en orina de 24 horas y niveles de anticonvulsivantes, esto último si el paciente está en tratamiento ${ }^{29}$.

- Finalmente, en la evaluación nutricional se debe interrogar por el tipo de dieta que tenía el paciente y posibles alergias. Se debe incluir en el examen físico: el peso, la estatura, el espesor del pliegue cutáneo y el índice de masa corporal basal. Si es posible se realiza una calorimetría indirecta (evaluación del metabolismo basal) y una densitometría ósea ${ }^{28}$.

Es fundamental que durante este proceso de evaluación se realice educación dirigida a los padres sobre la dieta y la importancia que exista una estricta adherencia para obtener los mejores resultados.

\section{Inicio de la dieta y control paraclínico intrahospitalario}

El inicio de la dieta debe estar supervisado por un neurólogo y un nutricionista que estén entrenados en el manejo de pacientes con $\mathrm{DC}^{9}$.

La dieta tradicionalmente se inicia de forma intrahospitalaria para facilitar una educación adecuada a los padres, tener un mayor control de posibles complicaciones (hipoglucemia, acidosis, náuseas, vómito, deshidratación) y para evaluar la posibilidad de intolerancia durante el periodo de ayuno inicial de 12-48 horas, que busca un control más rápido de las convulsiones ${ }^{30,31}$.

La meta es lograr tener niveles de beta-hidroxibutirato entre 2-5 nM después de 7 días de iniciar la $\operatorname{dieta}^{24}$. Posterior al periodo de ayuno, se introduce lentamente el consumo de calorías hasta lograr una relación 4:1 de grasas-carbohidratos y proteínas. Luego de lo anterior, el paciente permanece 3 a 4 días hospitalizado más para ser dado de alta ${ }^{28}$.

Durante la hospitalización se debe monitorizar la glucosa sérica cada 6-8 horas, si se encuentran valores menores de $30 \mathrm{mg} / \mathrm{dl}$ se debe suministrar una pequeña cantidad de jugo de naranja. Se deben medir los cuerpos cetónicos en orina cada 24 horas, para monitorizar la meta de beta-hidroxibutirato ${ }^{29}$. En múltiples estudios se ha demostrado que el periodo de ayuno inicial no tiene efecto sobre el control de la epilepsia a largo plazo, y solo incrementa el riesgo de hipoglucemia y cambios del estado de conciencia en los pacientes ${ }^{32}$, por esto se recomienda abolir el periodo de ayuno en niños menores de 2 años o en niños mayores que no tengan buen apetito 0 tengan múltiples comorbilidades, limitaciones en el habla para expresar fatiga o náuseas ${ }^{29}$. En este grupo de pacientes se podría considerar iniciar la dieta de forma ambulatoria y más progresiva ${ }^{29}$.

\section{Ajustes en dieta cetogénica}

La DC tradicional tiene una relación 4:1 de grasas-carbohidratos y proteínas, esto genera una restricción de calorías de entre un $80-90 \%$ de lo ideal para el peso ${ }^{29}$. En algunas condiciones, por intolerancia a la dieta, efectos adversos o alteraciones en el crecimiento pondoestatural, se puede disminuir la ratio a 3:1, sin embargo, estas reducciones podrían disminuir la eficacia de la dieta en los primeros tres meses ${ }^{29}$. 
Con el objetivo de mejorar la tolerancia de la dieta sin disminuir su eficacia han aparecido una gran cantidad de variantes de la dieta. Entre ellas se encuentran:

- Dieta de triglicéridos de cadena mediana: la mayor cantidad de grasa proviene de un suplemento de aceite, el cual genera más cuerpos cetónicos, tiene una mejor absorción y se metaboliza directamente en el hígado. Esto produce una disminución de la necesidad de grasas y, por lo tanto, un aumento de las proteínas y carbohidratos de dieta ${ }^{33}$.

- Dieta modificada de Atkins: se puede iniciar extrahospitalaria y se usa una relación 1:1 o 2:1. Se va realizando una restricción gradual de los carbohidratos y un aumento de las grasas, sin periodo de ayunas ${ }^{34}$.

- Dieta con un bajo índice de glucosa: se restringen los carbohidratos a 40-60 gramos al día y no se restringen las proteínas ni los líquidos, se limita al consumo de carbohidratos con índice de glucosa bajo $(<50)^{34}$.

\section{Efectos adversos a corto y largo plazo}

Todos los pacientes que estén recibiendo DC deben estar con suplementos multivitamínicos que incluyan calcio, vitamina $D$ y citrato de potasio para disminuir los posibles efectos adversos de la dieta ${ }^{28}$. Se ha visto en los estudios que la frecuencia de efectos adversos de la dieta suelen ser mínimos ${ }^{10}$.

A corto plazo se puede evidenciar síntomas gastrointestinales (12-50\%) como diarrea, constipación y reflujo gastroesofágico. Puede haber aparición de dislipidemia (14-59\%), episodios de hipoglucemia (25\% de pacientes en la semana inicial), hiperuricemia (2-26\%), hipoproteinemia, hipomagnesemia $(5 \%)$, hiponatremia, hepatitis y acidosis metabólica $(2-5 \%)^{10}$.

A largo plazo se pueden evidenciar alteraciones del crecimiento, deficiencia de carnitina, nefrolitiasis, deficiencia de selenio y enfermedad ósea como osteopenia, osteoporosis y aumento del riesgo de fracturas ${ }^{28}$.

Otros efectos adversos menos comunes son: pancreatitis, deficiencia de hierro, diátesis hemorrágica, cambios vasculares, cardiomiopatías y prolongación del $Q T^{10}$.

\section{Seguimiento ambulatorio}

En los pacientes que estén en tratamiento con la dieta se debe hacer un seguimiento estricto con el fin de detectar posibles efectos adversos o intolerancia y también verificar su eficacia. El control ambulatorio incluye una evaluación clínica cada tres meses durante el primer año por parte de Neurología, Pediatría y
Nutrición. Las evaluaciones deben ser más frecuentes en niños con alto riesgo nutricional ${ }^{29}$.

En estas visitas se debe monitorizar el peso, la altura y el consumo de calorías, estos deben ajustarse según la ganancia o pérdida de peso del niño. También se recomienda realizar un control electroencefalográfico cada tres meses, principalmente en niños menores de un año o en pacientes con encefalopatía epiléptica. Se sugiere evaluar el estado neurocognitivo cada 6 a 12 meses $^{29}$.

El seguimiento paraclínico se realiza cada tres meses y debe incluir hemoleucograma, perfil metabólico, perfil lipídico y carnitina ${ }^{28}$.

De forma ambulatoria los padres deben evaluar los cuerpos cetónicos en orina dos veces al día y la glucemia cada 12 horas en los primeros tres meses $^{28}$.

\section{Información para la familia}

Varias razones se exponen a las familias para su participación en la DC, demostrando que los beneficios superan los $\operatorname{costos}^{35}$. Se debe manifestar que su efectividad se evidencia en la capacidad de reducir las convulsiones en más del $50 \%$ de los casos, mejorando la calidad de vida del paciente y de los cuidadores $^{36}$. Se debe instruir sobre su metodología de uso y sus posibles efectos adversos ${ }^{5,37,38}$. Durante el inicio de la dieta en el hospital, los padres reciben clases del mecanismo de acción de la dieta, preparación de los alimentos y manejo de las complicaciones y las enfermedades, además de compartir calendarios que den información de la actividad convulsiva ${ }^{39-41}$.

El nutricionista debe enseñar las recetas y las reglas de la dieta que incluye no recibir infusiones intravenosas de glucosa ${ }^{42,43}$. Se debe enseñar a los padres a calcular y pesar con la balanza de gramos todos los alimentos y las bebidas que van a consumirse, un pequeño error puede entorpecer los resultados ${ }^{44}$.

Los medicamentos que se administren por vía oral deben estar libres de carbohidratos y en casos de hipoglucemia sintomática (diaforesis, fatiga excesiva, estado mental alterado, taquicardia, taquipnea) los padres deben administrar 30-60 ml de jugo de naranja y deben acudir a Urgencias para la medición de glucosa en suero, aunque raramente se necesitan bolos intravenosos de glucosa para aumentar los niveles ${ }^{21}$.

\section{Suspensión de la dieta}

El método de interrupción se adapta a la respuesta del paciente a la dieta. Tradicionalmente se suspende 
luego de dos años de tratamiento, cuando esta ha sido efectiva (reducción de las convulsiones superior al $50 \%)^{45}$. En niños que tienen una reducción de más de un $90 \%$ de las convulsiones y que presentan pocos efectos secundarios, la dieta puede ser útil durante 6-12 años ${ }^{45,46}$. En niños que no tienen una respuesta efectiva durante 14 días desde el inicio, la dieta debería ser continuada al menos tres meses y luego suspenderse $^{16,45}$. Los niños que presenten efectos adversos graves como aumento marcado de triglicéridos, deshidratación y cetoacidosis diabética deberán suspender el tratamiento inmediatamente ${ }^{47-49}$. Estudios retrospectivos han encontrado recurrencia de las convulsiones al suspender la dieta entre un mes y dos años, independientemente de los niveles de cetosis urinaria ${ }^{50}$.

\section{Conclusión}

La DC logra disminuir las convulsiones en más de un $50 \%$, además de que maximiza el neurodesarrollo y la calidad de vida del niño y su familia. Para lograr dicha eficacia se deben tener en cuenta una serie de factores, como una adecuada asesoría inicial, un seguimiento interdisciplinario y una adecuada adherencia al tratamiento.

\section{Conflicto de intereses}

Los autores declaran que en este estudio no existen conflictos de interés relevantes.

\section{Fuentes de financiamiento}

No existió una fuente de financiamiento particular para este informe científico.

\section{Bibliografía}

1. Camfield $P$, Camfield $C$. Incidence, prevalence and aetiology of seizures and epilepsy in children. Epileptic Disord. 2015;17(2):117-23.

2. Rozo VM, Izquierdo A. Caracterización de los factores de riesgo de pacientes con epilepsia de difícil control en un hospital de cuarto nivel en Bogotá-Colombia. Acta Neurol Colomb. 2014;30(4):234-9.

3. Tejeiro-Martínez J, Barcenilla-Rodríguez B. Epidemiología de la epilepsia. Rev Neurol Clin. 2000;1:229-45.

4. Fisher RS, Acevedo C, Arzimanoglou A, Bogacz A, Cross JH, Elger CE, et al. ILAE official report: a practical clinical definition of epilepsy. Epilepsia. 2014;55(4):475

5. Araya-Quintanilla F, Celis-Rosati A, Rodríguez-Leiva C, Silva-Navarro C, Silva-Pinto $Y$, Toro-Jeria B. Efectividad de la dieta cetogénica en niños con epilepsia refractaria: revisión sistemática. Rev Neurol. 2016; 62:439-48.

6. Ramírez-Camacho A, Meavilla S, Catalán N, Gutiérrez A, Campistol J. Experiencia con la dieta cetogénica como tratamiento en la epilepsia refractaria. Rev Neurol. 2011;53:524-30.

7. Devinsky O. Patients with refractory seizures. N Engl J Med. 1999; 340(20): 1565.

8. Luat AF, CoyleL, Kamat D. The ketogenic diet: A practical guide for pediatricians. Pediatr Ann. 2016;45(12):e446-50.
9. Sell E. Alternativas terapéuticas en epilepsia refractaria: Evolución y pronóstico de la cirugía de epilepsia, dieta cetogénica, y estimulación cerebral. Acta Neurol Colomb. 2008;24(1):34-9.

10. Kossoff EH, Zupec-Kania BA, Amark PE, Ballaban-Gil KR, Christina Bergqvist AG, Blackford R, et al. Optimal clinical management of children receiving the ketogenic diet: recommendations of the International Ketogenic Diet Study Group. Epilepsia. 2009;50(2):304.

11. Yudkoff M, Daikhin Y, Melo TM, Nissim I, Sonnewald U, Nissim I. The ketogenic diet and brain metabolism of amino acids: relationship to the anticonvulsant effect. Ann Rev Nutr. 2007;27:415-30.

12. Juge N, Gray JA, Omote H, Miyaji T, Inoue T, Hara C,et al. Metabolic control of vesicular glutamate transport and release. Neuron. 2010; 68:99-112.

13. Lefevre F, Aronson N. Ketogenic diet for the treatment of refractory epilepsy in children: A systematic review of efficacy. Pediatrics. 2000; 105(4):E46.

14. Nodli DR, Kuroda MM, Carroll J, Koenigsberger DY, Hirsch LJ, Bruner HJ, et al. Experience with the ketogenic diet in infants. Pediatrics. 2001; 108:129-33

15. Guzel O, Uysal U, Arslan N. Efficacy and tolerability of olive oil-based ketogenic diet in children with drug-resistant epilepsy: A single center experience from Turkey. Eur J Paediatr Neurol. 2019;23(1):143-51.

16. Wirrell E, Eckert S, Wong-Kisiel L, Payne E, Nickels K. Ketogenic diet therapy in infants: Efficacy and tolerability. Euro J Paediatric Neurology. Pediatr Neurol. 2018;82:13-8.

17. Kossoff EH, Pyzik PL, McGrogan JR, Vining EP, Freeman JM. Efficacy of the ketogenic diet for infantile spasms. Pediatrics. 2002;109(5):780-3.

18. Bough KJ, Rho JM. Anticonvulsant mechanisms of the ketogenic diet. Epilepsia. 2007;48(1):43.

19. Bough KJ, Wetherington J, Hassel B, Pare JF, Gawryluk JW, Greene JG, et al. Mitochondrial biogenesis in the anticonvulsant mechanism of the ketogenic diet. Ann Neurol. 2006;60(2):223

20. Danial NN, Hartman AL, Stafstrom CE, Thio LL. How does the ketogenic diet work? Four potential mechanisms. J Child Neurol. 2013;28(8):1027-33.

21. Lee PR, Kossoff EH. Dietary treatments for epilepsy: management guidelines for the general practitioner. Epilepsy Behav. 2011;21(2): 115-21.

22. Nangia S, Caraballo RH, Kang HC, Nordli D, Scheffer IE. Is the ketogenic diet effective in specific epilepsy syndromes? Epilepsy Res. 2012; 100(3):252-7.

23. Armeno M, Caraballo R, Vaccarezza M, Alberti MJ, Ríos V, Galicchio S, et al. Consenso nacional sobre dieta cetogénica. Rev Neurol. 2014; 59(5):213-23.

24. Berg AT, Kelly MM. Defining intractability: comparisons among published definitions. Epilepsia. 2006;47(2):431.

25. van der Louw E, van den Hurk D, Neal E, Leiendecker B, Fitzsimmon G, Dority $L$, et al. Ketogenic diet guidelines for infants with refractory epilepsy. Eur J Paediatr Neurol. 2016;20(6):798-809.

26. Kossoff EH, Hartman AL. Ketogenic diets: new advances for metabolism-based therapies. Curr Opin Neurol. 2012;25(2):173-8.

27. Neal EG, Chaffe H, Schwartz RH, Lawson MS, Edwards N and Fitzsimmons $\mathrm{G}$. The ketogenic diet for the treatment of childhood epilepsy: a randomised controlled trial. Lancet Neurol. 2008;7(6):500-6.

28. Veggiotti P, Burlina A, Coppola G, Cusmai R, De Giorgis V, Guerrini R, et al. The ketogenic diet for Dravet syndrome and other epileptic encephalopathies: An Italian consensus. Epilepsia. 2011;52(2):83-9.

29. Kossoff EHW, Shields WD. Non pharmacologic care for patients with Lennox-Gastaut syndrome: Ketogenic diets and vagus nerve stimulation. Epilepsia. 2014;55(4):29-33.

30. Lima PA, Sampaio LP, Damasceno NR. Neurobiochemical mechanisms of a ketogenic diet in refractory epilepsy. Clinics (Sao Paulo). 2014; 69(10):699-705.

31. Freeman J, Veggiotti P, Lanzi G, Tagliabue A, Perucca E. The ketogenic diet: from molecular mechanisms to clinical effects. Epilepsy Res. 2006;68(2):145-80.

32. Taub KS, Kessler SK, Bergqvist AG. Risk of seizure recurrence after achieving initial seizure freedom on the ketogenic diet. Epilpesia. 2014; 55(4):579-83.

33. Augustin K, Khabbush A, Williams S, Eaton S, Orford M, Cross JH, et al. Mechanisms of action for the medium-chain triglyceride ketogenic diet in neurological and metabolic disorders. Lancet Neurol. 2018;17(1):84-93.

34. Gano LB, Patel M, Rho JM. Ketogenic diets, mitochondria, and neurological diseases. J Lipid Res. 2014;55(11):2211-28.

35. Keene D. A Systematic review of the use of the ketogenic diet in childhood epilepsy. Pediatr Neurol. 2006;35:1-5.

36. Baby N, Vinayan KP, Pavithran N, Grace Roy A. A pragmatic study on efficacy, tolerability and long term acceptance of ketogenic diet therapy in 74 South Indian children with pharmacoresistant epilepsy. Epilepsy. 2018;58:41-6.

37. Nabbout R, Mazzuca M, Hubert P, Peudennier S, Allaire C, Flurin V, et al. Efficacy of ketogenic diet in severe refractory status epilepticus initiating fever induced refractory epileptic encephalopathy in school age children (FIRES). Epilepsia. 2010;51(10):2033-7. 
Rev Mex Neuroci. 2019;20

38. Singh RK, Joshi SM, Potter DM, Leber SM, Carlson MD, Shellhaas RA Cognitive outcomes in febrile infection-related epilepsy syndrome treated with the ketogenic diet. Pediatrics. 2014;134; e1431.

39. Jung DE, Kang $\mathrm{HC}$, Kim HD. Long-term outcome of the ketogenic diet for intractable childhood epilepsy with focal malformation of cortical development. Pediatrics. 2008;122:e330-3.

40. Villaluz MM, Lomax LB, Jadhav T, Cross JH, Scheffer IE. The ketogenic diet is effective for refractory epilepsy associated with acquired structural epileptic encephalopathy. Dev Med Child Neurol. 2018;60(7): 718-23.

41. Casey JC, McGrogan J, Pillas D, Pyzik P, Freeman J, Vining EP. The implementation and maintenance of the ketogenic diet in children. $\mathrm{J} \mathrm{Neu}$ rosci Nurs. 1999;31(5):294-302.

42. Freeman JM, Kelly MT, Freeman JB. The epilepsy diet treatment: an introduction to the ketogenic diet. $2^{\text {nd }}$ ed. Demos publications; 1996.

43. Smith G, Wagner JL, Edwards JC. Epilepsy update, part 2: nursing care and evidence-based treatment. Am J Nurs. 2015;115(6):34-44 quiz 45-6.
44. Joshi SM, Singh RK, Shellhaas RA. Advanced treatments for childhood epilepsy: beyond antiseizure medications. JAMA Pediatr. 2013;167(1):76-83.

45. Patel A, Pyzik PL, Turner Z, Rubenstein JE, Kossoff EH. Long-term outcomes of children treated with the ketogenic diet in the past. Epilepsia. 2010;51(7):1277-82

46. Caraballo R, Vaccarezza M, Cersósimo R, Rios V, Soraru A, Arroyo H, et al. Long-term follow-up of the ketogenic diet for refractory epilepsy: multicenter Argentinean experience in 216 pediatric patients. Seizure. 2011;20(8):640-5.

47. Thammongkol S, Vears DF, Bicknell区Royle J, Nation J, Draffin K, Stewart KG, et al. Efficacy of the ketogenic diet: which epilepsies respond?. Epilepsia. 2012;53(3)

48. Freeman JM, Kossoff EH, Hartman AL. The ketogenic diet: one decade later. Pediatrics. 2007:119(3):535-43.

49. Kossoff EH, McGrogan JR. Worldwide use of the ketogenic diet. Epilepsia. 2005;46:280-2.

50. Martinez CC, Pyzik PL, Kossoff EH. Discontinuing the ketogenic diet in seizure-free children: recurrence and risk factors. Epilepsia. 2007;48(1):187-90. 